\title{
Conoscopy of chiral smectic liquid crystal cells
}

\author{
Jang-Kun Song, ${ }^{1}$ J. K. Vij, ${ }^{1, *}$ and B. K. Sadashiva ${ }^{2}$ \\ ${ }^{1}$ Department of Electronic and Electrical Engineering, Trinity College, University of Dublin, Dublin 2, Ireland \\ ${ }^{2}$ Raman Research Institute, C. V. Raman Avenue, Sadashivanagar, Bangalore 560 080, India \\ *Corresponding author: jvij@tcd.ie
}

Received November 15, 2007; revised March 12, 2008; accepted April 11, 2008; posted May 8, 2008 (Doc. ID 89794); published June 30, 2008

\begin{abstract}
The conoscopic method for investigating the optical properties of a liquid crystal cell is studied with the aim of determining the effects of the approximations used in the calculation on the results. We confirm that the chiral liquid crystal cell forming a helical structure can be regarded as a single biaxial plate for analyzing the conoscopic image only if the helical pitch is less than several multiples of the wavelength of light. This approximation implies that the square of the refractive index along a direction is averaged over all the layers. An incorrectly chosen value for one of the principal refractive indices to be used in the analysis of the conoscopic data can lead to an incorrect conclusion, especially for the case when the wavelength dispersion of the refractive index is neglected. A thicker cell and a longer wavelength of the incident light can minimize these limitations of the conoscopic method. We propose a novel simulation method to find the molecular distribution in a liquid crystal cell based on the average-refractive-index approximation and the conoscopic data. This is shown to be a fast, more efficient, and useful method for estimating the director distributions. (c) 2008 Optical Society of America

OCIS codes: $000.2170,120.4640,160.1585,160.3710,180.1790,260.1440$.
\end{abstract}

\section{INTRODUCTION}

The conoscopic method is an optical technique used to investigate an optically transparent specimen placed in a cone of the converging rays of light placed within the crossed polarizers (see Section 2 for details). This technique has been extensively used for investigating the properties of liquid crystals. The method is also widely used in the liquid crystal display industry for measuring the viewing angle properties of displays and determining the image quality from oblique directions of the incidence of light. For the latter, various commercial measurement systems have been developed [1]. Scientifically, the method has been found to be an extremely powerful tool for investigating and modeling the various properties of liquid crystals. The method is used in determining the eigen refractive indices of the refractive index ellipsoid of liquid crystal cells. Here a cell is considered as an optically single biaxial plate having three principal refractive indices $n_{o 1}, n_{o 2}$, and $n_{e}$. Galerne [2] obtained the biaxiality $\left(\Delta n=n_{o 2}-n_{o 1}\right)$ and the apparent tilt angle $(\Theta$, the angle between the directions of $n_{e}$ and the normal to the cell) of achiral liquid crystal material in its various phases using conoscopic experiments. Gorecka et al. [3] carried out similar experiments by applying an electric field across ferroelectric liquid crystal cells, where helical structures may be formed. Fujikawa et al. [4] observed the dynamic behavior of a conoscopic image by using a CCD camera, and Song and coworkers contributed significantly to enlarging the observable range of the tilt angle of chiral smectics by developing the tilted conoscopic system $[5,6]$.

As stated above, conoscopy has been used for various scientific and technological applications including those for determining the refractive index ellipsoid [2-6], in estimating the molecular distribution [5-8], and in identifying the liquid crystalline phases $[3,4,9]$. Smectic $C$ liquid crystal phases such as $\mathrm{Sm}^{*}, \mathrm{Sm}_{A}^{*}, \mathrm{Sm}_{\gamma}^{*}$, and $\mathrm{AF}^{*}$ phases have a layered structure; the thickness of a layer is of the order of a few nanometers. Here, $\mathrm{SmC} C^{*}$ represents the ferroelectric phase, $\mathrm{Sm}_{A}^{*}$ and $\mathrm{AF}^{*}$ are the antiferroelectric phases having a periodicity of two and four layers, respectively, and $\mathrm{SmC}_{\gamma}^{*}$ is a ferrielectric liquid crystalline phase having the periodicity of three layers; (*) indicates the molecular chirality, which implies that the relevant phase has a helical structure with a periodicity ranging from a few tens of nanometers to a few tens of micrometers. The average direction of the molecules in smectic layers is tilted from the normal to the layers by the tilt angle, $\theta$, and can be located on the surface of an imaginary cone having a cone angle of $2 \theta$ as shown in Fig. 1. The azimuthal angle of the average molecular direction from the $x$ axis in the smectic layer in Fig. 1 is represented as $\phi$. The relative angles $\left(\phi_{i}-\phi_{i+1}\right)$ between the tilting directions of the neighboring layers vary according to the phase. These angles are as follows: Almost 0 for the $\mathrm{Sm} C^{*}$ phase, $\pi$ for the $\mathrm{Sm} C_{A}^{*}$ phase, and either zero or $\pi$ for the $\mathrm{SmC}_{\gamma}^{*}$ and $\mathrm{AF}^{*}$ phases. Due to the substructure of a few layers periodicity and of a longer helical structure, each phase shows a special characteristic behavior during the field induced unwinding process of the helix; hence the values of the biaxiality $\left(\Delta n=n_{o 2}-n_{o 1}\right)$ and the apparent tilt angle $(\Theta)$ under low electric fields have been used for identifying the phases. For example, the melatopes parallel to the applied field in conoscopic images have been used for assigning the phase as $\operatorname{Sm}_{\gamma}^{*}[3,4,9]$, but it has been reported that such a qualitative identification technique can in some cases lead to incorrect conclusions $[7,10]$. Melatopes parallel to the applied field can appear not only in a $\mathrm{Sm} C_{\gamma}^{*}$ phase but also in $\mathrm{SmC}^{*}$ and $\mathrm{SmC}_{\alpha}^{*}$ phases $[5,7,10]$, and therefore, we cannot state with certainty that the directions of the melatopes in the cono- 


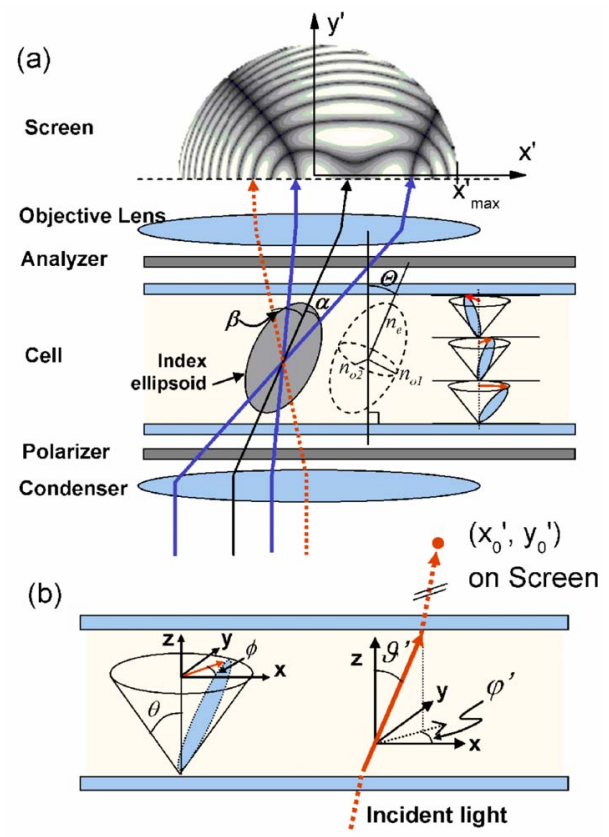

(c)

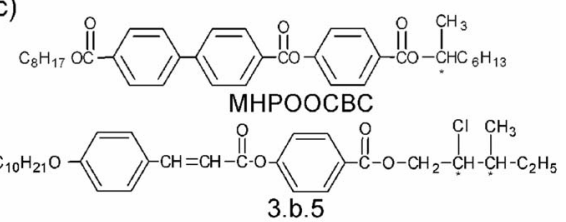

Fig. 1. (Color online) Schematic for conoscopy: (a) setup, (b) a cell, and (c) the chemical structure of liquid crystals used. The $x^{\prime}-y^{\prime}$ plane is the screen plane, and the $x, y$, and $z$ coordinate system is for the cell. The directions of two optic axes thick lines (blue online), a dark line dashed lines (red online) and the tilt direction of the refractive index ellipsoid (black thin line) are required for calculating the biaxiality and the apparent tilt angle $\Theta$. Homeotropically aligned smectic $C$ liquid crystals have a layered structure. In each smectic layer, the average direction of molecules is tilted from the normal to the cell by $\theta$, called the tilt angle, and the tilting direction, $\phi$, varies vertically from layer to layer and forms a helical structure. $\alpha$ and $\beta$ are the angles of the optic axes and the $N$ th dark ring from the major axis of the index ellipsoid, respectively ( $N$ can be arbitrararily chosen, and here, $N=2$ ). The directional vector $\left(\vartheta^{\prime}, \varphi^{\prime}\right)$ in the cell corresponds to the point $\left(x_{0}^{\prime}, y_{0}^{\prime}\right)$ on the screen. $\vartheta^{\prime}$ and $\varphi^{\prime}$ are the polar and azimuthal angles of the direction of the light in the cell. The chemical name of the laboratory named compound (RRI, Bangalore) 3.b.5 is [2s,3s] $4^{\prime}$-(2-Chloro3-methlypentyloxycarbonyl) phenyl trans $4^{\prime \prime}$-n-decyloxycinnamate.

scopic image relative to the low external electric field can correctly identify the phase. For a clear identification of the phase, it is required to calculate the exact values of the biaxiality and the apparent tilt angle and to accurately estimate the molecular distribution in a cell. Therefore, an accurate and a quantitative method of the analysis of conoscopy is required. Furthermore, conoscopy has mostly been used as a qualitative auxiliary technique in the study of liquid crystals. Furthermore, a quantitative analysis has rarely been made so far. This is presumably due to the fact that despite a simplicity involved in carrying out the conoscopic experiments and obtaining the data, analysis of the results and their meaningful interpretations are quite complicated. Furthermore, the quantitative method for analyzing the conoscopic image contains a number of approximations, and their validity and the errors in obtaining the results have not been explored as yet.

In this paper, we investigate the method used in the analysis of the conoscopic data and find that two approximations exist in the analysis: (i) A single-biaxial-plate assumption in helical phases and (ii) a choice of one of the principal refractive indices with a view to using the technique. We also suggest a better utilization of the technique of conoscopy for the study of liquid crystals and develop an effective simulation method to determine the molecular distribution from the measured conoscopic results based on using the average-refractive index method.

\section{CONOSCOPY AND A VERIFICATION OF THE APPROXIMATIONS USED IN ITS ANALYSIS}

\section{A. Experiment and Analysis}

We briefly explain the conoscopic experiment and the procedure for the analysis of the conoscopic images [3,6,11]. A schematic is shown in Fig. 1 . The conoscopic method consists of two cross polarizers and the use of at least two optical lenses. The screen and the sample cell are placed at the focal planes of the objective lens. The light passing through the sample along each direction gives rise to a distribution of the intensity on the screen, independent from all other directions. Each point on the image projected on the screen (i.e., the $x^{\prime}-y^{\prime}$ plane in Fig. 1) normal to the plane of the paper corresponds to a certain direction of the incident light into the cell, and the direction is determined by a simple geometric calculation. The direction of light outside the cell can be represented as the polar angle $\vartheta^{\prime}$ and the azimuthal angle $\varphi^{\prime}$, where $\vartheta^{\prime}$ is the angle of incidence with respect to the normal to the cell and $\varphi^{\prime}$ is the angle of the projection of light on the cell plane with respect to the $x$ axis. The center point of the image corresponds to the direction $\left(\vartheta^{\prime}, \varphi^{\prime}\right)=(0,0)$, and the maximum observable polar angle is determined by the value of numerical aperture $\left[(\mathrm{NA})=\sin \vartheta^{\prime}\right.$ in air] of the lens, and therefore, $\vartheta_{\max }^{\prime}$ at the boundary of the image is given as $\vartheta_{\text {max }}^{\prime}=\sin ^{-1}(\mathrm{NA})$. Hence, a particular point $\left(x^{\prime}, y^{\prime}\right)=\left(x_{0}^{\prime}, y_{0}^{\prime}\right)$ on the screen corresponds to the direction $\left(\vartheta^{\prime}, \varphi^{\prime}\right)$ $=\left(\tan ^{-1}\left[\tan \left(\vartheta_{\max }^{\prime}\right)\left(x_{0}^{\prime 2}+y_{0}^{\prime 2}\right)^{1 / 2} / x_{\max }^{\prime}\right], \tan ^{-1}\left(y_{0}^{\prime} / x_{0}^{\prime}\right)\right)$, where $x_{\max }^{\prime}$ is the radius of the image. The intensity projected on the screen reflects the phase difference or the retardation that the light experiences in passing through the sample. Therefore, the two-dimensional patterns on the screen contain the information about the refractive index ellipsoid of the sample. This can be extracted from the conoscopic pattern through a series of calculations. Conoscopic observations can be made by using a specially designed conoscopic instrument or by using a polarizing microscope equipped with a Bertrand lens. A Bertrand lens in a polarizing microscope is placed between the objective and the ocular lenses for making the observations of the socalled directional image or the conoscopic image. The conoscopic images given in this paper were obtained by using an optical polarizing microscope (Olympus BX-52). We used lenses with large NA for both the objective (Olympus, LM PlanFI; 50, 0.5 NA) and the condenser 
(Instech; 0.65 NA) for maximizing the observable range. Homeotropically aligned liquid crystal cells each of $100 \mu \mathrm{m}$ in cell thickness were prepared by using a homeotropic alignment agent, carboxylatochromium complexes (chromolane). The liquid crystals used are (R) (1-methylheptyloxycarbonyl)phenyl-4' -carboctyloxybiphenyl-4-carboxylate (MHPOOCBC), 3.b.5, and Felix018. Chemical structures of MHPOOCBC and 3.b.5 are shown in Fig. 1. Felix018 is a commercial ferroelectric liquid crystal mixture synthesized by Hoechst AG, Germany, and the chemical structure of it is unknown.

Conoscopic images captured using a digital camera (Leica DFC480) mounted onto the microscope head were fed to a computer for analysis. The conoscopic image produced by a biaxial plate consists of concentric elliptic dark rings and the two parabolic dark lines as shown in Fig. 1, where only a half of the conoscopic image is shown. The vertices of the parabolas correspond to the optic axes of the biaxial plate. Hence, one can obtain approximate information about the refractive index ellipsoid that qualitatively mimics the cell from the shape and the direction of the parabolas.

A brief procedure for obtaining the biaxiality and the apparent tilt angle of the liquid crystalline compound confined in the cell is detailed here. The two optic axes and a dark point on the concentric elliptic rings are used for calculating the biaxiality and the apparent tilt angle of the liquid crystal in the cell. The angle of the incident light outside the cell for the two optic axes (thick lines, blue online), for a dark point (dashed line, red online) and for the apparent tilt direction (black thin line), can be obtained by the simple geometric calculation explained above, and the directional information can easily be transformed to the approximate directions of light inside the cell using Snell's law and an approximate value of the refractive in$\operatorname{dex}(\tilde{n}=1.5)$ in the geometry shown in Fig. 1 [10]. In this way, the apparent tilt angle, $\Theta$, is directly obtained from the calculation. Similarly, angles of the optic axes and the $n$th dark point from the major axis of the refractive index ellipsoid, which are denoted as $\alpha$ and $\beta$, respectively, are obtained. Incident light is separated into two paths inside the biaxial cell; the path is determined by the two eigen refractive indices for the direction. When the light propagates in the plane including the two optic axes of the refractive index ellipsoid of the cell, the two eigen refractive indices are $n_{o 2}$ and $n_{o 1}$, if $n_{o 2}>n_{o 1}$. However, if $n_{o 2}<n_{o 1}$, the two eigen refractive indices are $n_{o 1}$ and $n_{o 2^{\prime}}$. The eigen refractive index $n_{o 1^{\prime}}$ is found as

$$
n_{o 1^{\prime}}=\left[\left(\frac{\cos \zeta}{n_{e}}\right)^{2}+\left(\frac{\sin \zeta}{n_{o 1}}\right)^{2}\right]^{-1 / 2}
$$

where $\zeta$ is the angle of the light path from the direction of $n_{e}$ inside the cell. In the second case, $n_{o 2}$ and $n_{o 1}$ are replaced by $n_{o 2^{\prime}}$ and $n_{o 2}$. Almost zero transmission of the incident light corresponding to the dark point on the screen means that the phase difference of the two beams that have been split by the birefringent medium after passing through it is an integral multiple of the wavelength $\lambda$. For the optic axes and the dark lines on the screen, therefore, one can write a simple equation as

$$
n_{o 1^{\prime}} l_{o 1^{\prime}}-n_{o 2} l_{o 2}=m \lambda \quad(m=0,1,2 \cdots),
$$

where $l_{o 1^{\prime}}$ and $l_{o 2}$ are the geometrical path lengths of the two split beams and are found as $l_{o 1^{\prime}, 02}$ $=d \cdot n_{o 1^{\prime}, o 2} / \sqrt{n_{o 1^{\prime}, o 2}^{2}-\sin ^{2}(\vartheta)}$, where $d$ is the cell thickness and $\vartheta$ is the polar angle of the incident light outside the cell with respect to the normal to the cell. Hence, Eq. (2) can be rewritten as

$$
d\left[\frac{n_{o 1^{\prime}}^{2}}{\sqrt{n_{o 1^{\prime}}^{2}-\sin ^{2} \vartheta}}-\frac{n_{o 2}^{2}}{\sqrt{n_{o 2}^{2}-\sin ^{2} \vartheta}}\right]=m \lambda \quad(m=0,1,2 \cdots) .
$$

In Eqs. (1) and (3), $d$ and $\lambda$ are obtained from the experimental conditions and $\vartheta$ is measured from the conoscopic image. When $\zeta=\alpha$, that is, the light propagates along either of the two optic axes, $m=0$, and when $\zeta=\beta$, that is, the light propagates along the direction corresponding to the $N$ th dark line, $m=N$ (in Fig. $1, N=2$ ). Each of these conditions produces one set of equations from Eqs. (1) and (3), and these equations can be used to find the principal refractive indices of the ellipsoid. By assuming one of the values of the principal refractive indices (for example, $n_{o 1}=1.5$ ), the other two principal refractive indices are numerically obtained from Eqs. (1) and (3). The obtained values of the principal refractive indices are used to recalculate $\alpha, \beta$, and $\Theta$, and in order to improve the accuracy of the obtained values, the same procedure is iterated several times when the calculated principal refractive indices quickly converge. $\Theta$ is the apparent tilt angle of the ellipsoid shown in Fig. 1. Finally, the biaxiality is calculated as $\left(n_{o 2}-n_{o 1}\right)$. This is an explicit method to find the biaxiality, but Eqs. (1)-(3) can be further simplified to obtain analytic equations, which enable us to analytically find the other two principal refractive indices by using further approximations so that $l_{o 1^{\prime}} \approx l_{o 2}$ in Eq. $(2)[3,11$.

In these calculations, two approximations are made. First, the cell is considered as a single biaxial plate as shown in Fig. 1, and second, one of the values of the principal refractive indices is assumed, for example, $n_{o 1}=1.5$. We examine these two assumptions in Subsections 2.B and 2.C.

If the optical properties of a sample are known, the conoscopic image can be conversely simulated. The $4 \times 4$ matrix method $[12,13]$ is useful for this purpose. When a polarized light passes through a single or multilayered biaxial plate, one can accurately calculate the polarization and the amplitude of the transmitted and reflected lights using the $4 \times 4$ matrix method. In particular, when a sample is placed between the two polarizers, the transmittance of the incident light can be calculated for any direction of the incident light. For simulating the conoscopic image, one needs to decide the resolution of the image and the range of the polar angle. Once the resolution and the range are determined, the screen is divided into a number of square grids to represent the resolution. Positional information of each point of the grids on the screen is changed into the polar and azimuthal angles of the light. By using the $4 \times 4$ matrix method, the transmittance is calculated for each direction of light and is plotted for each point of the screen corresponding to the direction of 
the incident light. In this way, one can obtain a two dimensional conoscopic pattern for a given stack of biaxial plates.

\section{B. Single-Biaxial-Plate Approximation}

Since Gorecka et al. [3] measured the biaxiality and the apparent tilt angle as a function of the applied field using conoscopy for a chiral smectic liquid crystal in its various phases, such as $\mathrm{SmC}, \mathrm{SmC}_{A}^{*}$, and $\mathrm{SmC}_{\gamma}^{*}$ phases, this method adopting more or less the same procedure has frequently been used by a number of authors [2-8]. In those papers, the cells were considered as single-biaxial plates. The cells of achiral $\operatorname{Sm} C$ and $\operatorname{Sm} A$ phases in reality are single biaxial plates, and the tilt angle and biaxiality are reasonably defined. However, the cell containing the chiral smectic in its various phases is not a single biaxial plate, and strictly speaking, the biaxiality cannot be defined in the cell having a helical structure. We pose the fundamental question: What is the meaning of the biaxiality obtained using conoscopy in chiral smectic phases? In chiral smectic phases, each layer has its own refractive index ellipsoid, and the directions of the principal axes gradually vary from layer to layer. Hence, the biaxiality and the apparent tilt angle estimated from conoscopic experiments can only represent the average properties of the various layers.

In the approximation of the isotropic local field, the principal refractive indices $\left(n_{i}\right)$ of the uniform media can be written as $[14,15]$

$$
\frac{n_{i}^{2}-1}{\left\langle n^{2}\right\rangle+2}=\frac{4 \pi N\left\langle\alpha_{i i}\right\rangle}{3}
$$

where the average square of refractive index $\left\langle n^{2}\right\rangle$ $=\left(n_{1}^{2}+n_{2}^{2}+n_{3}^{2}\right) / 3$. The polarizability component $\left\langle\alpha_{i i}\right\rangle$ is the average value along the direction of the principal refractive index $n_{i}$, and $N$ is the number of molecules per unit volume. The principal refractive indices of the averagerefractive-index ellipsoid consisting of $m$ layers can be expressed from Eq. (4) as

$$
\begin{aligned}
n_{i}^{2} & =\frac{1}{m} \sum_{j=1,2, \ldots, m}\left[\frac{4 \pi}{3} N\left\langle\alpha_{i i}\right\rangle_{j}\left(\left\langle n^{2}\right\rangle+2\right)+1\right] \\
& =\frac{4 \pi N\left(\left\langle n^{2}\right\rangle+2\right)}{3}\left[\frac{1}{m_{j=1,2, \ldots, m}} \sum_{i i}\left(\left\langle\alpha_{i i}\right\rangle_{j}\right)\right]+1 \\
& =\frac{4 \pi N\left\langle\alpha_{i i}\right\rangle\left(\left\langle n^{2}\right\rangle+2\right)}{3}+1 .
\end{aligned}
$$

Thus, Eq. (5) is identical to Eq. (4). This implies that the average-refractive index can be defined by averaging the polarizability component over the layers. By assuming

$$
n_{i j}^{2} \approx \frac{4 \pi}{3} N\left\langle\alpha_{i i}\right\rangle_{j}\left(\left\langle n^{2}\right\rangle+2\right)+1,
$$

where $n_{i j}$ is the refractive index value along the $i$ th direction of the $j$ th layer, we can define the average refractive index along the arbitrary direction $(\hat{\varphi})$ of the layered structure as

$$
n_{\hat{\varphi}}^{2} \approx\left\langle n_{\hat{\varphi} j}^{2}\right\rangle_{j=1,2, \ldots, m}
$$

Equation (7) defines the average value of the refractive index over all the layers, and this may not always be valid. However, if only the single-biaxial-plate approximation is applicable, Eq. (7) is valid, that is, the singlebiaxial-plate approximation is identical to the averagerefractive-index approximation given by Eq. (7). If the thickness of a single period in a periodic structure is much less than the wavelength of light, incident light will experience the average refractive index, and therefore Eq. (7) is valid. However, if the thickness of a single period is larger than the wavelength, Eq. (7) may not be valid. For example, when the helical pitch of a smectic $C$ liquid crystal is larger than the wavelength of the incident light, the average-refractive-index approximation is not supposed to be valid, and the liquid crystal cells cannot be considered as a single biaxial plate. In this case, the cell should be considered as a stack of a number of biaxial plates, and each layer should be separately accounted for. However, the approximation in the literature has been used even for the phase having a long helical structure without confirming its validity. For example, the structures of $\mathrm{SmC}_{A}^{*}$ and $\mathrm{SmC}_{\gamma}^{*}$ phases of 4-(1methylheptyloxycarbonyl)phenyl 4'-octyloxybiphenyl-4carboxylate (MHPOBC), a prototype antiferroelectric liquid crystalline compound, were determined by observing the conoscopic images $[3,4,9]$, and the pitch of MHPOBC is reported to be approximately $670 \mathrm{~nm}$ in the $\mathrm{SmC}_{A}^{*}$ phase and much larger in the $\mathrm{SmC}_{\gamma}^{*}$ phase. In the $\mathrm{SmC}_{\gamma}^{*}$ phase, the selective reflection band does not appear for wavelengths shorter than $2.5 \mu \mathrm{m}$, which means that the pitch is longer than $(2.5 / n) \mu \mathrm{m}$ (where $n$ is the refractive index of liquid crystal). However, the biaxiality and the apparent tilt angle of MHPOBC in the $\mathrm{SmC}_{\gamma}^{*}$ phase were measured, and the $\mathrm{SmC}_{\gamma}^{*}$ phase was characterized based on a single-biaxial-plate approximation.

Thus, in general it is required to test the validity of the average-refractive-index method for helical structures in order to arrive at the correct conclusions. First, we shall test the validity of the approximation in a twisted nematic (TN) mode. The TN mode has been used in a majority of commercial liquid crystal displays, and the optical properties of the $\mathrm{TN}$ mode have been investigated in greater detail [16]. Hence, the TN mode is suitable for testing the validity of the approximation. The transmittances of the TN mode are calculated as a function of the cell thickness as shown in Fig. 2 using the two methods: The $4 \times 4$ matrix method [12,13] and the averagerefractive-index method defined in Eq. (7). The $4 \times 4$ matrix method gives an accurate value of the transmittance, whereas the average-refractive-index method provides an approximated value. By comparing the results from these two methods, we can find the range of validity of the average-refractive index method. Since the TN mode has $90^{\circ}$ twisted directors, the pitch $p$ is proportional to the cell thickness $d(p=4 d)$. The results from the two methods show good agreement with each other for a range of short helical pitches as shown in Fig. 2 while the difference between these significantly and sharply increases for $p>8 \mu \mathrm{m}$. Thus, the average-refractive-index approximation is not valid for structures with longer helical pitches, 


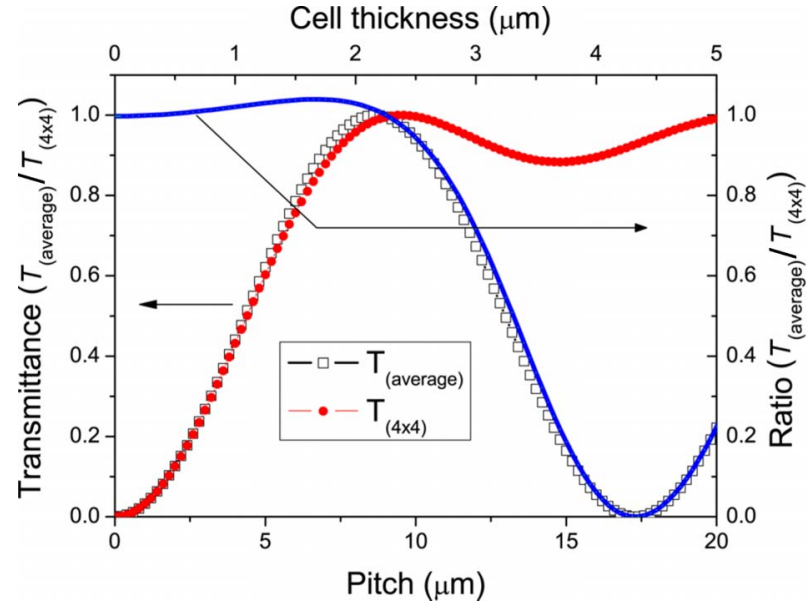

Fig. 2. (Color online) Simulated transmittances as a function of pitch or the cell thickness in TN mode by using the $4 \times 4$ matrix method $\left(T_{(4 \times 4)}\right)$ and average-refractive-index method $\left(T_{\text {(average) }}\right)$. Simulation condition: $90^{\circ}$ twist nematic, $n_{o}=1.5$, and $n_{e}=1.7$.

but we can see that the method is reasonably applicable for the samples having short helical pitches up to several times the wavelength of light.

Now, we shall confirm the validity of this method for the smectic phases with a longer helical pitch. For this, we obtained an accurate conoscopic image by using the $4 \times 4$ matrix simulation and compare it with the result simulated by using the single-biaxial-plate approximation. A uniform helical structure produces a symmetric shape of the conoscopic image, where both the apparent tilt angle and the biaxiality are zero. Therefore, for obtaining nonzero values of these two parameters, we need to use a deformed helical structure. Since a helical structure is a periodic structure, the azimuthal angle $\phi$ of the average direction of molecules, defined in Fig. 1, in a deformed helical structure under the application of field can be written using the Fourier expansion as [17]

$$
\phi(z) \approx q z+\phi_{0} \sin q z+\phi_{1} \sin (2 q z)+\cdots,
$$

where the wave vector $q$ and the pitch $p$ are related to each other as $q=2 \pi / p$. Considering that the purpose of this simulation is to verify the average-refractive-index approximation by using an assumed helical structure of the smectic liquid crystalline compound, we do not need to use a number of terms in Eq. (8); first we shall use up to the second term and the director distribution when $\phi_{0}$ $=-1$. The simulation conditions are $40 \mu \mathrm{m}$ cell thickness, $4 \mathrm{~nm}$ layer thickness, $\theta=25^{\circ} ; n_{e}=1.7, n_{o}=1.5$, and $\lambda=450$ and $650 \mathrm{~nm} . \theta$ is the smectic layer tilt angle (molecular tilt angle). The simulation result is shown in Figs. 3(a) and 3(b), where the first two images in 3(a) and 3(b) are obtained using the average-refractive-index approximation, and the others are obtained using the $4 \times 4$ matrix simulation method. The number in the inset in Fig. 3 for each conoscopic image corresponds to a value of the helical pitch. As shown in Figs. 3(a) and 3(b), the simulation result using the average-refractive-index approximation finds good accord with those obtained using the $4 \times 4$ matrix method for short helical pitches. However, as the pitch increases, the optic axes become obscured, and the shapes of the melatopes get deformed. The deformation (a) simulation $(\lambda=650 \mathrm{~nm})$

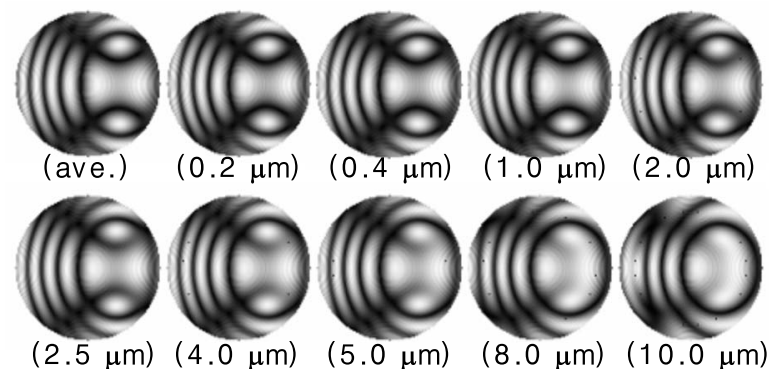

(b) simulation $(\lambda=450 \mathrm{~nm})$

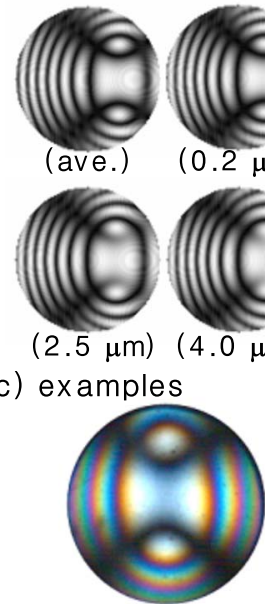

$(0.4 \mu \mathrm{m})$

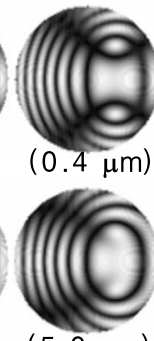

$(5.0 \mu \mathrm{m})$
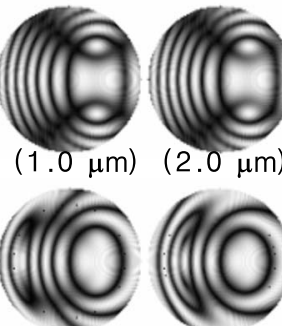

$(8.0 \mu \mathrm{m})$

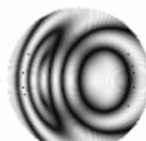

$(10.0 \mu \mathrm{m})$

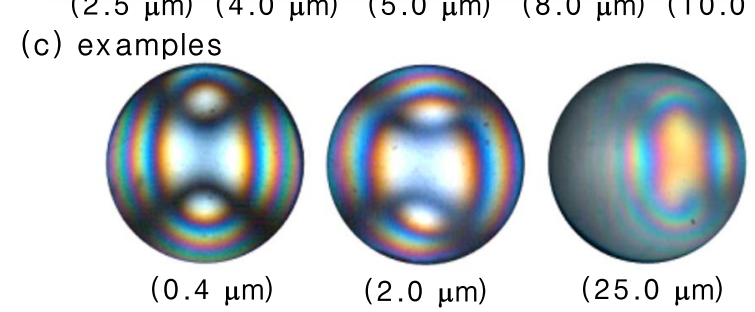

Fig. 3. (Color online) (a), (b) Simulated conoscopic images and (c) experimentally obtained examples. The first images indexed by (ave.) in (a) and (b) were obtained by the average-refractive method, and all the others were from the $4 \times 4$ matrix method. The numbers in parentheses are the length of the pitch for the simulation condition of the $4 \times 4$ matrix method. The first two images in (c) were taken by using pure $(R)$ MHPOOCBC $(p \sim 0.4 \mu \mathrm{m})$ and a racemic mixture $(p \sim 2 \mu \mathrm{m})$ of $(R)$ MHPOOCBC $65 \%$ and $(S)$ MHPOOCBC $35 \%$, respectively, and the last one was taken by using Felix018 ( $p \sim 25 \mu \mathrm{m})$.

for the incident light of wavelength $\lambda=450 \mathrm{~nm}$ is much greater than for $\lambda=640 \mathrm{~nm}$. The transmittances along the line passing the two optic axes are plotted in Fig. 4. The optic axes are marked by the arrows (blue online), and the transmittances at the optic axes points should be zero in the ideal case, but the transmittances at the points increase with an increase in the helical pitch. The positions of the optic axes and dark lines also move with the pitch. These give rise to an error in the value of the biaxiality using this method for the analysis of conoscopy. We can see that the shift of the optic axes and the dark lines is much greater for $\lambda=450 \mathrm{~nm}$ than for $\lambda=650 \mathrm{~nm}$.

It is very interesting that the simulated conoscopic images using the $4 \times 4$ matrix method do not significantly change for $p<5 \mu \mathrm{m}$ at $\lambda=650 \mathrm{~nm}$ and for $p<3 \mu \mathrm{m}$ at $\lambda=540 \mathrm{~nm}$ from those simulated using the averagedrefractive index method. This means that the average refractive index approximation is valid up to a pitch of roughly 7 times $\lambda$. It has been reported [18] that when a nonhomogeneous medium containing microstructures is approximated as a homogeneous medium, this approximation is valid for a periodic nanoarray structure placed perpendicular to the direction of light if the periodicity of 

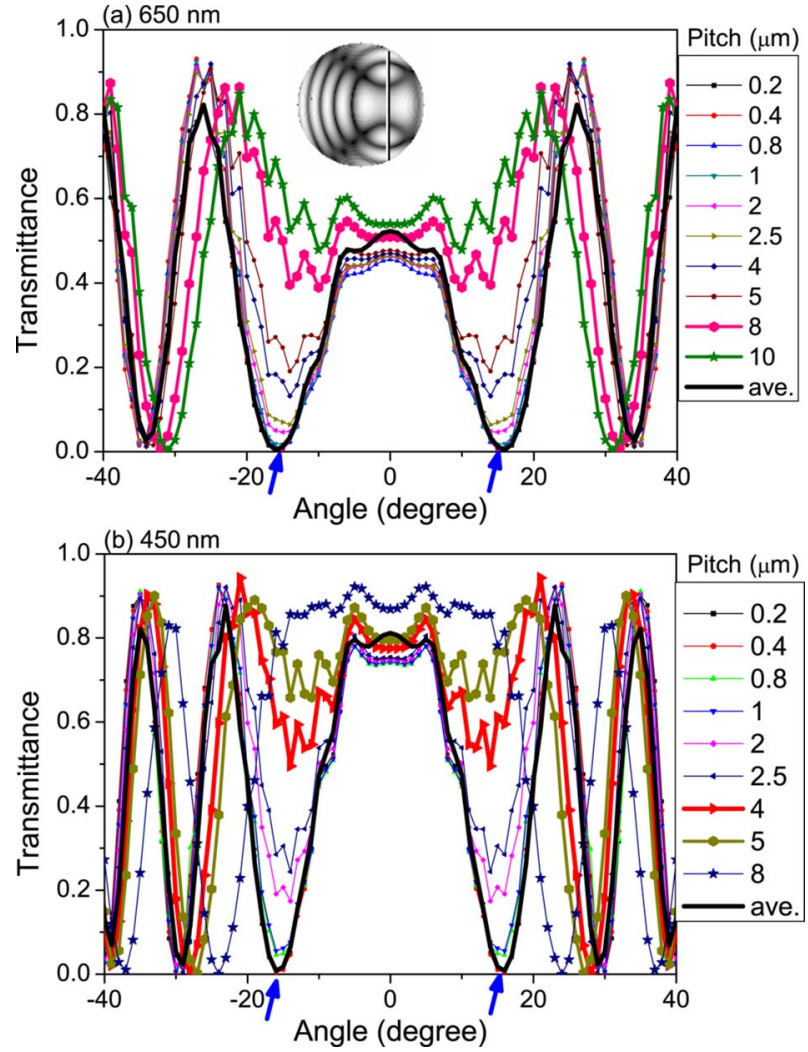

Fig. 4. (Color online) Simulated transmittance as a function of the angle of light with respect to $n_{e}$ along the line passing the two optic axes in the simulated conoscopic images in Fig. 3 [see the inset image in (a)]. The pitches in the insets are the simulation condition for the $4 \times 4$ matrix method, and (ave.) indicates the average-refractive-index method. Points marked with arrows (blue online) are optic axes.

the structure is a few times less than the wavelength of light. However, as shown above, in the helical structure of liquid crystal cells, if the periodic structure is placed parallel to the direction of light, the homogenization approach using the average-refractive index approximation is valid up to a much longer helical pitch than the wavelength of light.

To date, it has been accepted [8] that the use of a shorter wavelength is a more accurate conoscopic method in liquid crystals because it has the advantage of showing up more rings in the image, and this may thus reduce the errors arising from misassignment of the optic axes. However, the use of a longer wavelength leads to lower errors induced by the average-refractive-index approximation and also enlarges the observable range of the pitch of the materials that can be investigated using this technique. Figure 3(c) experimentally shows observed conoscopic images for different helical pitch values, where a similar tendency predicted from the simulation results is observed.

\section{Assumption of Fixing One of the Principal Refractive Indices}

Usually, the main advantage of the conoscopic technique is a determination of the biaxiality $\left(n_{o 2}-n_{o 1}\right)$ but not of determining the absolute values of the refractive indices. By taking an approximate value for one of the principal

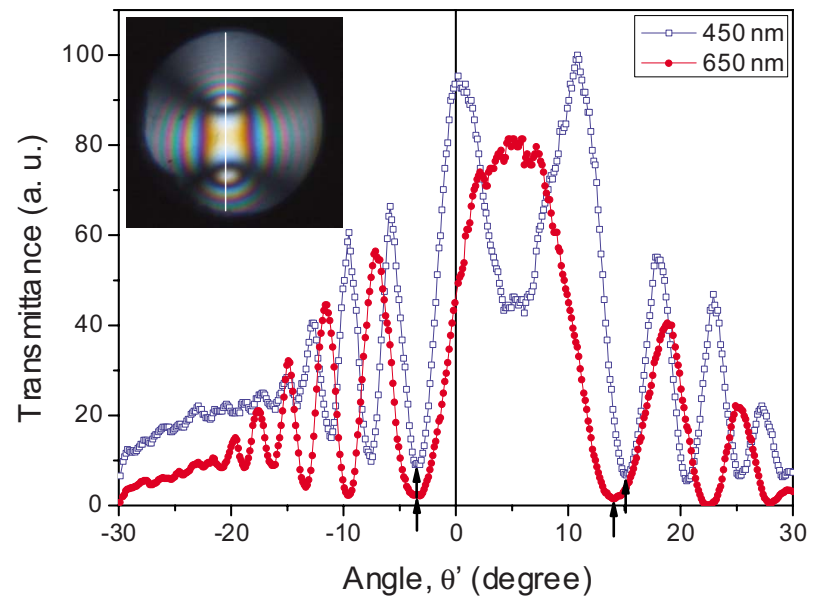

Fig. 5. (Color online) Transmittance along the curve passing between the two optic axes at wavelengths (red and blue online) obtained by using 3.b.5 (experimental conditions: $E=200 \mathrm{~V} / \mathrm{mm}$, $40^{\circ} \mathrm{C}$ ). The chemical name of the laboratory named compound (RRI, Bangalore) 3.b.5 is [2s,3s] $4^{\prime}$-(2-Chloro3-methylpentyloxycarbonyl) phenyl trans 4 "-n-decyloxycinnamate.

refractive indices, the other two values can be reasonably calculated. As was already pointed out by Gorecka et al. [3], when $n_{o 1}$ is incorrectly chosen as 1.45 , a value less than the real value of say 1.5, an error in the biaxiality caused by this incorrectly chosen value was found to be less than $1 \%$, which can be neglected. However, an error in the apparent tilt angle found from conoscopy is rather large compared to that for the biaxiality. The light path in the cell can be calculated using Snell's law

$$
\vartheta=\sin ^{-1}\left[\sin \left(\vartheta^{\prime}\right) / n_{0}\right]
$$

where $n_{0}$ is the refractive index of light normal to the direction of propagation in an anisotropic medium and $\vartheta$ and $\vartheta^{\prime}$ are the angles of the incident light inside and outside the cell, respectively. The tilt angle is also calculated using Eq. (9). According to Eq. (9), an error in the tilt angle caused by the same incorrectly chosen $n_{o 1}$ is found to be $3.5 \%-4 \%$, which is rather large. The error caused by this effect can also be a problem when conoscopy is carried out on a sample for various wavelengths of light. Figure 5 shows a typical conoscopic image of a ferroelectric liquid crystal, 3.b.5, and a halogen lamp as the source of light. The transmittance curves along the curve across the two optic axes for blue color (online, $450 \mathrm{~nm}$ ) and (red color online, $650 \mathrm{~nm}$ ) wavelengths clearly show different positions of the optic axes. One of the optic axes close to the center of the image is in the same position in the two curves while the second away from the center appears in different positions. According to Fig. 5, the apparent tilt angle found appears to be dispersive with $\lambda$. This may be thought to have been induced by the local field effect [10]. We measured the apparent tilt angle as a function of the applied field using a different method with a planar cell (data not shown here). However we could not find any change in the apparent tilt angle as a function of $\lambda$. The apparent tilt angle dispersion caused by the local field effect may be too small to detect using the usual optical method. Thus, the shift of the optic axis observed in Fig. 5 does not arise from the dispersion of the apparent tilt angle. The shift actually arises from Eq. (9), where $n_{o 1}$ 
varies with $\lambda$ and hence $\vartheta$ is found to be dispersive with $\lambda$, which is not the case in reality. We therefore find that the effect of choosing an incorrect value of $n_{o 1}$ gives rise to incorrect results for the apparent tilt angle, especially when a number of wavelengths of the incident light are used. The dispersion of the position of the optic axis in the conoscopic images can be used to determine the dispersion of the refractive index of liquid crystals.

\section{ESTIMATION OF THE MOLECULAR DISTRIBUTION USING THE CONOSCOPIC DATA}

\section{A. Simulation Method to Estimate the Molecular Distribution}

As explained in Section 1, we can estimate the director distribution in a cell from the results of a conoscopic experiment. In earlier papers [2-4,9], the distribution was qualitatively and intuitively estimated. For example, in the $\mathrm{Sm}_{A}^{*}$ phase, the tilting direction ( $\phi$ in Fig. 1) of molecules in smectic layers is supposed to align parallel to the applied field, and the angle difference $\left(\phi_{i}-\phi_{i+1}\right)$ between the adjacent layers is almost $\pi$, that is, the tilting directions are in opposite directions to each other. This results in a large positive biaxiality and a small apparent tilt angle as previously explained [9]. Later, Suwa and coworkers $[7,8]$ estimated the director distribution in the deformed helical structure of a ferroelectric liquid crystal by using the $4 \times 4$ matrix simulation, where a more precise quantitative estimation was tried for the first time. Though their method of using the $4 \times 4$ matrix simulation is quite interesting, it showed apparent limitations.

Basically, the procedure of determining the director distribution from the conoscopic data is the same as the procedure of finding the optimized fitting parameters from the experimental data. In this procedure, the experimental data are compared with the simulation results by varying the fitting parameters in order to find the optimized condition, where the differences between the simulation and the experiment are minimized. For this fitting, a proper model for the director distribution in the cell needs to be found. This model may vary depending on the application, liquid crystalline phase, and the cell structure. Fourier expansion shown in Eq. (8) can be a simple but good model, or a theoretical molecular distribution function can be obtained by minimizing the total free energy containing the elastic and the electric energy [6]. Suwa et al. [8] used a much more simplified model for the director distribution. Once the model is established, we should decide what physical properties are compared between the model and the experiment to optimize the fitting parameters. A direct comparison of the conoscopic images obtained by experiment and simulation creates various difficulties because the two-dimensional transmittance patterns in the conoscopic images are significantly complicated and contain narrow rings, which turns the quantitative comparison into a difficult task. Therefore, the biaxiality and the apparent tilt angle are used for drawing a comparison [6,8] since the biaxiality and the apparent tilt angle actually contain most of the information about the conoscopic image. Then, a considerable iteration is required for finding the optimum condition.
For this iteration procedure, the approach using the $4 \times 4$ matrix method has limitations. One of these limitations is that the $4 \times 4$ matrix calculation requires a long time to iterate many times $[12,13,19]$, although the calculation time has significantly been reduced by simplifying the matrix method [20]. Note that the simulation of the conoscopic image requires one to produce a twodimensional conoscopic pattern, and each point of the two-dimensional image requires the $4 \times 4$ matrix calculation to be carried out independent of any other point. Another limitation lies in the difficulty of extracting the biaxiality information from the simulated conoscopic images. The $4 \times 4$ matrix calculation gives only the transmittance information, that is, the conoscopic image. However, the physical property to compare with the experimental data is the biaxiality and the apparent tilt angle. Hence, we need to extract the biaxiality from the simulated conoscopic image. Due to the complexity of the conoscopic image, however, it is not easy to automatically find the positions of the optic axes. As a result, it is difficult to automatically iterate the simulation.

Meanwhile, the method using the average-refractive index defined by Eq. (7) can be used for solving this problem. As shown in Subsection 2.B, the average-refractiveindex approximation is valid for a relatively wide range of helical pitch values and directly gives values of the apparent tilt angle and the biaxiality. This means that the average-refractive-index method is suitable for carrying out iteration in the simulation and is much faster than the $4 \times 4$ matrix method. Therefore, we can easily find the optimum director distribution using the experimental values of the apparent tilt angle and the biaxiality.

\section{B. Simulation of the Deformed Helical Structure}

As an example of the estimation of the molecular distribution along the cell thickness direction, i.e., along the normal to the smectic layers, we shall simulate a deformed helical structure of a common ferroelectric liquid crystalline material. We used a racemic mixture of $(R) \mathrm{MHPOOCBC} 65 \%$ and $(S) \mathrm{MHPOOCBC} 35 \%$, where $(R)$ and $(S)$ indicate the handedness of chiral group $(R$, rectus or right; $S$, sinister or left). The racemic mixture showed a typical continuous unwinding process over a wide range of applied fields and is suitable for this simulation while pure $(R)$ MHPOOCBC showed an irregular nonhomogeneous helical fracture [6]. It is difficult to find a molecular distribution function for the irregular structure. The conoscopic image in Fig. 6 was obtained under the application of $27 \mathrm{~V} / \mathrm{mm}$ at $70^{\circ} \mathrm{C}$. From the conoscopic image, the following values are found: Biaxiality $=-0.00101$ and the apparent tilt angle $=14.7^{\circ}$. By applying a larger field (of say $250 \mathrm{~V} / \mathrm{mm}$ ), the conoscopic image in the helical unwound state was obtained. Since the tilting directions are the same for all of the smectic layers in the unwound state, the optical properties of the unwound cell are identical to the properties of a single smectic layer. In this way, we find that the principal refractive indices of each layer are 1.65, 1.501, and 1.50 , respectively, and the tilt angle $\theta$ is found to be $27^{\circ}$. Based on these data, we carry out a simulation to find the molecular distribution of a deformed helical structure for an electric field of $27 \mathrm{~V} / \mathrm{mm}$. We use the Fourier expan- 

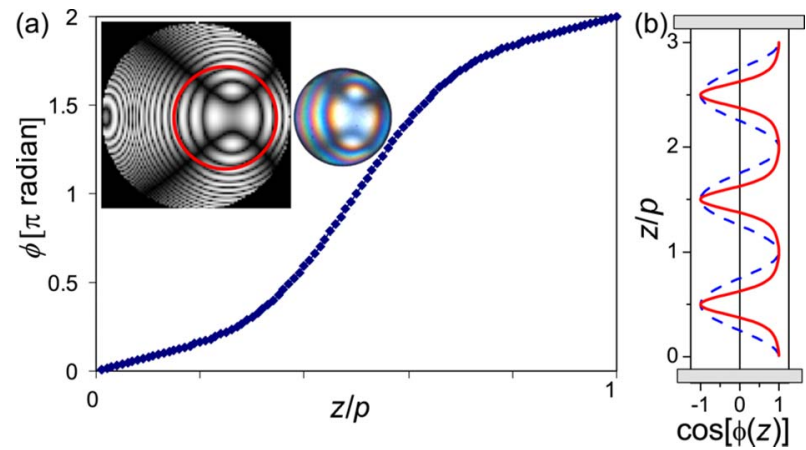

Fig. 6. (Color online) (a) Director distribution estimated from the conoscopic image using a racemic mixture of MHPOOCBC (right inset image), and the reproduced conoscopic image from the calculated director distribution (left inset image). The experimental conoscopic image was obtained by using the tilted method, so the center point is shifted. The circle in the simulated conoscopic image denotes the observable range in the actual experiment. (b) Helix distortion by the applied field in the cell. Initial uniform helix (dashed line, blue online) changes to the distorted helical structure (solid line, red online) by the field.

sion as shown in Eq. (8) for the molecular distribution function along the cell thickness direction $z$. When we used up to the second term of Eq. (8), we could not perfectly satisfy both the biaxiality and the apparent tilt angle values experimentally obtained using any value of $\phi_{0}$. By using up to the third term of Eq. (8), we can find the optimized values of $\phi_{0}$ and $\phi_{1}$ for having the same tilt angle and the biaxiality as the experimental results, $\phi_{0}=-0.8775$ and $\phi_{1}=0.151$. The director distribution for this is shown in Fig. 6. Thus, the director distribution can simply be estimated by using the average-refractive-index approximation.

\section{CONCLUSION}

We investigate the procedure of analyzing the conoscopic image of chiral smectic liquid crystals, which basically assumes two approximations in the calculation procedure. We find that the single-biaxial-plate approximation for helical structures is valid for the pitch up to several times the wavelength of the incident light. A long wavelength of light from the source can enlarge the observable range of the helical pitch of the materials in the cell to be investigated. The arbitrarily chosen value of one of the principal refractive indices gives rise to the wavelength dispersion in the apparent tilt angle. This can make the refractive index dispersion appear as the dispersion of the apparent tilt angle with wavelength. We suggest a new simulation method for determining the molecular distribution function in liquid crystal cells of using the average-refractiveindex method, given by Eq. (7). This is a better, more efficient, and faster method for carrying out an automatic simulation that uses the iterative process.

\section{ACKNOWLEDGMENTS}

We thank A. Fukuda and K. Ishikawa for useful discussions and Nissan Chemical Industries (I. Kobayashi) for gifting the valuable materials. J. K. Song thanks Sam- sung Electronics Co., Ltd. for granting a leave of absence from Seoul. Science Foundation Ireland (SFI) is thanked for funding of the project 06/RF9/ENE039.

\section{REFERENCES AND NOTES}

1. One of the examples is the Conoscope manufactured by Autronic-Melchers GmbH, Karlsruhe, Germany.

2. Y. Galerne, "Refractive index measurements at a 2nd order smectic A to C phase transition," J. Phys. (Paris) 39, 1311-1316 (1978).

3. E. Gorecka, A. D. L. Chandani, Y. Ouchi, H. Takezoe, and A. Fukuda, "Molecular orientational structures in ferroelectric, ferrielectric and antiferroelectric smectic liquid crystal phases as studied by conoscope observation," Jpn. J. Appl. Phys. 29, 131-137 (1990).

4. T. Fujikawa, K. Hiraoka, T. Isozaki, K. Kajikawa, H. Takezoe, and A. Fukuda, "Construction of dynamic conoscope observation system using CCD camera and image processor," Jpn. J. Appl. Phys., Part 1 32, 985-988 (1993).

5. J. K. Song, A. D. L. Chandani, O. E. Panarina, A. Fukuda, J. K. Vij, V. Goertz, and J. W. Goodby, "Study of the SmC" phase in the Tokyo mixture by conoscopy using tilted cell," Ferroelectrics 344, 41-47 (2006).

6. J. K. Song, J. K. Vij, and I. Kobayashi, "Interlayer interactions and the dependence of biaxiality of the chiral smectic- $C^{*}$ phase on electric field in the helical unwinding process," Phys. Rev. E 75, 051705 (2007).

7. S. Suwa, H. Hoshi, Y. Takanishi, K. Ishikawa, H. Takezoe, and B. Zeks, "Helix unwinding process in a short-pitch ferroelectric liquid crystal mixture studied by conoscopy," Jpn. J. Appl. Phys., Part 1 42, 1335-1337 (2003).

8. S. Suwa, Y. Takanishi, H. Hoshi, K. Ishikawa, and H. Takezoe, "Helix unwinding process in the chiral smectic C phase of MHPOBC as observed by conoscopy," Liq. Cryst. 30, 499-505 (2003).

9. A. Fukuda, Y. Takanishi, T. Isozaki, K. Ishikawa, and H. Takezoe, "Antiferroelectric chiral smectic liquid crystals," J. Mater. Chem. 4, 997-1016 (1994).

10. J. K. Song, A. D. L. Chandani, A. Fukuda, J. K. Vij, I. Kobayashi, and A. V. Emelyanenko, "Temperature-induced sign reversal of biaxiality observed by conoscopy in some ferroelectric Sm- $C^{*}$ liquid crystals," Phys. Rev. E 76, 011709 (2007).

11. M. Born and E. Wolf, Principles of Optics (Cambridge U. Press, 1999), Chap. 14.4.

12. D. W. Berreman, "Optics in stratified and anisotropic media: 4×4-matrix formulation," J. Opt. Soc. Am. 62, 502-510 (1972).

13. P. Yeh, "Electromagnetic propagation in birefringent layered media,” J. Opt. Soc. Am. 69, 742-756 (1979).

14. D. Dunmur and K. Toriyama, Handbook of Liquid Crystals (Wiley-VCH, 1998), Vol. 1, Chap. 7, Sec. 3.

15. J. Li and S.-T. Wu, "Self-consistency of Vuks equations for liquid-crystal refractive indices," J. Appl. Phys. 96, 6253-6258 (2004).

16. I.-C. Khoo and S.-T. Wu, Optics and Nonlinear Optics of Liquid Crystals (World Scientific, 1993), Chap. 2.

17. J. K. Song, U. Manna, and J. K. Vij, "Mechanism of field induced unwinding of $\mathrm{SmC}^{*}$ helix and bias field dependence of dielectric permittivity and macroscopic spontaneous polarization," Europhys. Lett. 82, 26003 (2008).

18. I. Abdulhalim, "Simplified optical scatterometry for periodic nanoarrays in the near-quasi-static limit," Appl. Opt. 46, 2219-2229 (2007).

19. A. R. MacGregor, "Method for computing homogeneous liquid-crystal conoscopic figures," J. Opt. Soc. Am. A 7, 337-347 (1990).

20. I. Abdulhalim, "Analytic propagation matrix method for linear optics of arbitrary biaxial layered media," J. Opt. A, Pure Appl. Opt. 1, 646-653 (1999). 